Nereis. Revista Iberoamericana Interdisciplinar de Métodos, Modelización y Simulación
12

12

Valencia

(España)

\title{
El alginato y sus inmensas aplicaciones industriales
}

\author{
Alginate and its vast range of industrial usages
}

Fecha de recepción y aceptación: 13 de febrero de 2020, 22 de mayo de 2020

DOI: $10.46583 /$ nereis_2020.12.573

\author{
Alejandro Hurtado ${ }^{1}$, Rafael Selgas ${ }^{1}$ y Ángel Serrano-Aroca ${ }^{*}$ \\ ${ }^{1}$ Biomaterials and Bioengineering Lab. Centro de Investigación Traslacional San Alberto Magno. Universidad Católica de Valencia \\ San Vicente Mártir. \\ * Correspondencia: Universidad Católica de Valencia San Vicente Mártir. Centro de Investigación Traslacional San Alberto Magno. \\ Calle Guillem de Castro, 94. 46001 Valencia. España.E-mail: angel.serrano@ucv.es
}

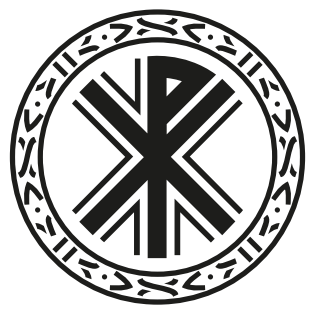

\section{RESUMEN}

El alginato es un biopolímero no tóxico, biodegradable, biocompatible, soluble en agua y renovable, extraído habitualmente de algas pardas o producido mediante cultivo microbiano. Este biomaterial está aprobado por la Food and Drug Administration (FDA) de Estados Unidos para su uso en humanos en ciertas aplicaciones biomédicas. Además, existe una amplia gama de tipos de alginato diseñados con propiedades diversas según la aplicación específica. En el presente trabajo de revisión se pretende mostrar las inmensas aplicaciones que ofrece este biopolímero y que siguen en crecimiento exponencial debido a sus excelentes propiedades químicas, físicas y biológicas. De este modo, se presentan aplicaciones actuales en áreas importantes como la industria farmacéutica, la industria química y textil, la industria gastronómica y de la congelación, la inmovilización biotecnológica de células y enzimas, la liberación controlada de biomoléculas, la industria biomédica y finalmente el análisis de su creciente uso en la bioimpresión 3D.

PALABRAS CLAVE: alginato, biomaterial, aplicaciones, biomedicina, bioimpresión 3D, industria, inmovilización, células, enzimas, liberación de biomoléculas, hidrogeles.

\begin{abstract}
Alginate is a non-toxic, biodegradable, biocompatible, water-soluble and renewable biopolymer, usually extracted from brown algae or produced by microbial culture. This biomaterial has been approved by the Food and Drug Administration (FDA) for human use in certain biomedical applications. In addition, there is a wide range of alginate types designed with different properties depending on the specific application. This review aims to show the multiple applications of this biopolymer, which continues to grow exponentially due to its excellent chemical, physical and biological properties. Thus, current applications are present in important areas such as the pharmaceutical industry, chemical and textile industry, gastronomic and freezing industry, biotechnological immobilization of cells and enzymes, controlled release of biomolecules and biomedical industry. Finally, we will discuss its increasing use in 3D bioprinting applications.
\end{abstract}

KEYWORDS: alginate, biomaterial, applications, biomedicine, $3 d$ bioprinting, industry, immobilization, cells, enzymes, biomolecule release, hydrogels. 


\section{INTRODUCCIÓN}

El alginato es un biopolímero no tóxico, biodegradable y renovable, extraído habitualmente de algas pardas pertenecientes a la clase filogénica Phaeophyceae u obtenido mediante cultivo microbiano [1]. Es un material aprobado por la Food and Drug Administration (FDA) de Estados Unidos para su uso en humanos en ciertas aplicaciones biomédicas, debido a sus excelentes propiedades de biocompatibilidad [2]. Existe una amplia gama de tipos de alginato con diferentes masas moleculares y proporciones entre grupos D-manurónico y L-gulurónico o modificados químicamente que le confieren una amplia gama de propiedades diferentes [3].

El presente trabajo de revisión pretende mostrar las inmensas aplicaciones de uno de los biopolímeros con las mejores propiedades químicas, físicas y biológicas existentes, el alginato. De este modo, podemos encontrar aplicaciones del alginato en infinidad de áreas diferentes como la industria farmacéutica, la industria química, la industria textil, la industria de la congelación, la industria gastronómica, la inmovilización de células y enzimas, la liberación controlada de biomoléculas, la industria biomédica y su más reciente uso en la bioimpresión 3D dentro de la medicina regenerativa.

\section{INDUSTRIA FARMACEÚTICA}

En la industria farmacéutica, el alginato puede utilizarse en su forma de sal como espesante para cremas o medicamentos tópicos [4]. La gran diversidad existente de tipos de alginato con diferentes masas moleculares y proporciones diferentes entre grupos D-manurónico y L-gulurónico es relevante en la industria farmacéutica para el desarrollo de nuevos productos [5]. Dependiendo del pH en el que se encuentre el alginato, este presenta una estructura diferente [6]. De este modo, a pH neutro el alginato se convierte en un gel que ayuda a la hidratación y liberación del contenido, y a pH ácido 3 el alginato se hincha. En la industria farmacéutica, los alginatos también sirven para aglutinar diversas sustancias y hacerlas visibles como comprimidos de uso oral [7].

\section{INDUSTRIA QUÍMICA Y TEXTIL}

El alginato presenta excelentes propiedades químicas para la formulación de detergentes para ropa [8], la producción de tintas de impresión textil [9] y para la coloración de tejidos [10]. Los alginatos son los espesantes más utilizados en la impresión de tejidos de celulosa [11]. Además de proporcionar una alta viscosidad, consiguen cambiar o alterar las propiedades reológicas y proporcionar un alto rendimiento hacia el color [12]. Este material, junto con los iones de cobre, es capaz de reducir considerablemente la actividad microbiológica, especialmente de la bacteria Gram-negativa Escherichia coli, en los tejidos formados por la lana [13]. El alginato se ha utilizado también junto a nanopartículas de $\mathrm{TiO}_{2}$ para producir tejidos antibacterianos y resistentes a múltiples lavados con agua [14]. La mezcla de fibras de alginato con gelatina en un medio con cloruro de calcio y etanol favorece la formación de una estructura fibrosa estable [15]. Además, se ha observado que las propiedades de las fibras de alginato en la industria textil mejoran cuando se introducen más iones de sodio en la composición de la estructura del polímero [16]. La mayoría de las fibras de alginato producidas en la 
industria química se destinan a usos médicos como transportadores de fármacos para la cicatrización tisular [17]. Finalmente, hay que destacar que con el fin de mejorar las propiedades del nylon en los tejidos, se utilizan enlaces cruzados de alginato [18]. El uso principal del alginato en la industria del papel es el dimensionamiento de la superficie. El alginato agregado al tamaño normal del almidón proporciona una película continua lisa y una superficie con menos pelusa. La resistencia al aceite de las películas de alginato proporciona un tamaño con mejor resistencia al aceite y mejora las propiedades a prueba de grasa. Se obtiene un brillo mejorado con tintas de alto brillo. Si se van a encerar papeles o cartones, el alginato del tamaño mantendrá la cera principalmente en la superficie. Proporcionan una mejor capacidad de recubrimiento que otros espesantes, especialmente en aplicaciones calientes de recubrimiento en máquina. Los alginatos también son excelentes formadores de película y mejoran la retención de tinta y la capacidad de impresión. La cantidad de alginato utilizada es generalmente del 5 al $10 \%$ del peso del almidón en el tamaño. El alginato también se usa en adhesivos de almidón para fabricar tableros corrugados porque estabiliza la viscosidad del adhesivo y permite el control de su velocidad de penetración. El $1 \%$ de alginato de sodio, basado en el peso del almidón utilizado, suele ser suficiente.

\section{INDUSTRIA DE LA GASTRONOMÍA Y DE LA CONGELACIÓN}

En esta área industrial, el alginato es especialmente importante desde la conservación de compuestos en helados hasta la de microorganismos probióticos microencapsulados [19]. Para mejorar la textura de un helado es necesaria una viscosidad adecuada. Esta propiedad tan importante de un buen helado puede conseguirse mediante una mezcla de gelatina y alginato de sodio entre otros compuestos [20]. Además, el uso de alginato en los helados mejora considerablemente, por ejemplo, el sabor de la vainilla en el hielo [21]. Si se desea retardar la cristalización se puede añadir isotiocianato de rodamina a una solución con alginato y sacarosa [22,23]. Además de en la industria de los helados, el alginato se utiliza también en la cocina para producir estructuras esféricas comestibles e hidrogeles con considerable resistencia [24].

La gastronomía molecular, que es una ciencia culinaria que pretende estudiar los mecanismos químicos que operan a nivel molecular cuando cocinamos, está en auge. Esta disciplina ha tenido un gran impacto debido a su atractivo, la calidad de los productos alimentarios producidos y la buena valoración realizada por los comensales [25].

Dentro de esta ciencia, la esferificación es un proceso en el que los alimentos licuados se mezclan con el alginato de sodio y se sumergen en una disolución que contiene iones de calcio que crean diferentes tipos de esferas [26]. De este modo, la esferificación con alginato se ha utilizado para encapsular distintos alimentos como el caviar, por ejemplo [27]. Una de las primeras aplicaciones de la técnica de esferificación se produjo cuando Ferran Adrià la puso en práctica en el Bulli en 2003 para hacer caviar de frutas y falsos ñoquis [28]. La esferificación inversa también se utiliza comúnmente. En este caso, los extractos de hojas de bardana mezclados con iones de calcio se dejan caer en una solución de alginato de sodio para producir microesferas de hidrogel de núcleo líquido [29]. Además, con esta técnica culinaria se consigue mejorar tanto el sabor como la textura y el alginato le confiere a la comida una textura y pegajosidad originales [30]. Hay estudios que se centran en la creación de 
esferas con alginato de agua dulce que se forman al cambiar ciertas propiedades físicas variando a diferentes concentraciones de sal de alginato [31].

Debido a sus exclusivas propiedades de aglutinamiento, espesamiento, gelificación, formación de película y estabilización, el alginato y sus muchos derivados tienen una larga historia de uso en alimentación e incluso como aglutinante en acuicultura. El espesamiento es útil en las salsas, jarabes, coberturas para helados, etc. Se requiere gelificación en postres y jaleas instantáneas de leche, crema de relleno de panadería y pasteles de frutas. Las propiedades coloidales generales son importantes cuando se agrega alginato de sodio al helado y cuando se usa alginato de propilenglicol (PGA) para estabilizar la espuma de cerveza o suspender los sólidos en las bebidas de frutas. La adición de alginato mejora la formación de hielo, haciéndolos no pegajosos [32].

\section{INMOVILIZACIÓN BIOTECNOLÓGICA DE CÉLULAS Y ENZIMAS}

La formación de esferas de alginato se realiza gota a gota de forma espontánea mediante la producción de una red iónica que fija las células en su interior [33]. Mediante la inmovilización se ha observado un aumento de la actividad enzimática, por ejemplo, en el cultivo cuando las células del Bacillus Stearothermophilus se fijan con alginato [34]. La inmovilización mediada por el polisacárido alginato proporciona un mayor nivel de actividad de enzimas como la quitinasa, una enzima que tiene grandes aplicaciones en biotecnología, principalmente para la producción de vacunas [35].

El primer y novedoso medio de gel que se utilizó para encapsular las enzimas fue un hidrogel de alginato [36]. Hoy en día se están explorando nuevas formas de inmovilización de células mediante alginato para la producción de diversas enzimas como la lipasa [37]. También se han fijado y encapsulado diferentes probióticos para obtener diversas ventajas: mayor productividad, mejor control de los procesos y mantenimiento de la capacidad estable y actividad biocatalítica durante un intervalo de tiempo más largo [38]. Una monocapa de alginato sobre una membrana de polisulfona comercial se utiliza para inmovilizar de forma no covalente diferentes tipos de enzimas [39]. La matriz formada por la adición de alginato permite aumentar el grado de cooperación enzima-sustrato [40]. Las enzimas se inmovilizan en el gel de alginato promoviendo nuevas estrategias, ya que permite que las enzimas no se filtren en el medio y el producto se libere al exterior [41]. La capacidad de la inmovilización para hacer reutilizables y estables las enzimas de alto coste ha significado un gran avance y por tanto ha atraído mucha atención en la industria de bioprocesado [42]. Es posible inmovilizar varias enzimas al mismo tiempo y la estructura química característica del alginato en forma de caja de huevos es clave para ello [43].

Actualmente se está estudiando el uso de alginato de cobre en lugar de alginato de calcio para la inmovilización enzimática, ya que se ha observado una mayor estabilidad y actividad enzimática [44]. Además, constituye un medio para el diseño de biorreactores utilizados en la limpieza de aguas contaminadas.

Los geles de alginato también se utilizan como vehículos de suministro de células en ingeniería tisular para permitir la localización de las células trasplantadas y controlar su destino [45]. Empresas como Sperm Vital utilizan el alginato para aumentar las probabilidades de éxito en la inseminación de ganado vacuno, ya que se permite una liberación controlada de los espermatozoides en el útero durante un período de tiempo más prolongado [46]. 


\section{INDUSTRIA BIOMÉDICA}

Dentro de la industria biomédica, el alginato presenta amplias aplicaciones en la industria oftalmológica. De este modo, el alginato se utiliza para la producción de lentes de contacto [47]. Además, se han desarrollado hidrogeles de colágeno que contiene microesferas de alginato con proteínas encapsuladas para la solución de problemas oculares [48]. Existe también la posibilidad de aplicar la deposición capa por capa, a base de quitosano y alginato, para guiar la liberación de diversos fármacos oftalmológicos [49].

En la industria dental, el alginato se aplica como uno de los materiales de impresión más importantes del mundo [50]. La pasta producida por el alginato se utiliza en esta área industrial para la fijación y fabricación de prótesis [51]. Además, se están estudiando nuevas composiciones de impresión de alginato con propiedades mejoradas tanto para uso dental como hospitalario [52]. Los alginatos se utilizan con frecuencia para mejorar la adhesión de las prótesis dentales [51]. El alginato también puede utilizarse en odontología en combinación con células madre para la fabricación de soportes porosos capaces de regenerar tejido $[53,54]$.

Asimismo el alginato se utiliza como material de impresión o molde para la producción de una copia a nivel completo del complejo óseo [55]. Se están introduciendo métodos para fabricar prótesis nasales septales personalizadas mediante la aplicación de moldes de alginato con detalles de perforación para producir una estructura clínica de silicona [56].

Hoy en día, se realizan impresiones completas de alginato para su uso facial posquirúrgico y se fijan para evitar que se desprendan [57]. En el campo de desarrollo de prótesis neuronales, se han fabricado andamiajes (o scaffolds en inglés) basados en alginato que incorporan células madre neuronales para favorecer la regeneración de dicho tejido [58]. Las ventajas de la aplicación de alginato en la industria protésica incluyen un coste reducido, una alta tolerabilidad para el paciente, una maleabilidad eficiente, un tiempo de uso reducido, una instrumentación y un procedimiento de ejecución muy sencillo y la posibilidad de detectar una impresión específica en un solo paso [59]. Con el tiempo, el alginato se ha representado como el primer material de impresión válido y seguro desde el punto de vista clínico.

Como biomaterial de origen natural, marino o microbiano, el alginato ha demostrado un gran potencial en la creación de materiales biomédicos y clínicos en terapias avanzadas como la ingeniería tisular, la biología celular, la liberación de fármacos y la generación de sustancias farmacéuticas debido a su eficiente actividad biológica y a su gran capacidad físico-química con un alto grado de control [60]. El alginato en la industria biomédica suele utilizarse como alginato ultrapuro, ya que la presencia de endoxinas y otros compuestos pueden producir efectos adversos en aplicaciones in vivo. De este modo, diversas empresas como DuPont ofrecen alginatos ultrapurificados bien caracterizados con diferentes viscosidades y contenidos de grupos $\mathrm{G}$ diversos para su uso en aplicaciones biomédicas y farmacéuticas. También ofrecen alginatos acoplados a péptidos que mejoran la adhesión celular y una gran variedad de alginatos estériles.

Sin embargo, el uso del alginato en biomedicina va más allá. Por un lado, este biopolímero juega un papel fundamental en la quelación de metales pesados en medicina. La toxicidad por metales pesados es un problema a nivel global que puede causar problemas de salud de diversos grados en diversos órganos y sistemas. Aunque hoy en día se utilizan quelantes intravenosos como el EDTA, lo cierto es que tienden a aparecer efectos secundarios debido que une indistintamente metales pesados 
y minerales esenciales para el correcto funcionamiento del organismo. La ventaja que suponen los alginatos de bajo peso molecular con respecto a los quelantes ampliamente utilizados se basa en que son capaces de unirse selectivamente a los metales pesados, dejando intactos a los elementos minerales esenciales [61]. Por otro lado, un estudio en bebés ha concluido que la utilización de alginato de magnesio reduce los síntomas persistentes de reflujo gastroesofágico en ellos, tanto en los lactantes como en los alimentados con fórmula [62].

Además, como ya comentamos anteriormente, los hidrogeles de alginato pueden ser muy útiles en la liberación controlada y localizada de fármacos y macromoléculas de bajo peso molecular para la regeneración tisular. La liberación de estas sustancias encapsuladas en polímeros de alginato permite una actuación más precisa y un tratamiento más sostenido en el tiempo [63]. Los factores de crecimiento liberados del alginato pueden promover o impedir la diferenciación celular [64], la proliferación y/o el crecimiento de tejidos [65] y la vascularización. La industria biomédica ha crecido mucho debido a aplicaciones innovadoras del alginato, como por ejemplo la empresa AlgiPharma, que ha desarrollado un tratamiento basado en oligómeros de alginato para tratar a pacientes con fibrosis cística [66].

\section{BIOIMPRESIÓN 3D}

La bioimpresión 3D, o bioprinting en inglés, es una técnica que permite la fabricación directa de un tejido vivo u órgano completo artificial. Esto se lleva a cabo mediante la combinación de biomateriales, células y factores de crecimiento. Para ello, se realiza un paso previo de preimpresión en el que se utilizan modelos de tomografía computarizada óptica y 3D, y datos de resonancia magnética del tejido u órgano que se pretende copiar [67]. A partir de estas, se dispensan, capa por capa, los bloques de construcción multicelular con bioenlaces y se escalan para la fabricación de la construcción final. El resultado es la producción automática y reproducible de tejidos vivos y funcionales en 3D que son herramientas más reproducibles y realistas a la hora de llevar a cabo experimentos con fármacos o estudios toxicológicos e incluso para realizar trasplantes [68]. Sin embargo, no cualquier material es susceptible de ser usado para este fin, ya que debe cumplir ciertos requisitos de biocompatibilidad y propiedades estructurales y/o mecánicas básicas, para lo cual los más recomendados son los hidrogeles, más concretamente los de alginato [69]. Los hidrogeles basados en alginato son los más utilizados en el campo de la bioimpresión 3D debido a que este es un polímero de origen natural, biodegradable, no tóxico y no inmunogénico, además de que posee un precio relativamente económico. No obstante, el alginato también presenta sus inconvenientes, ya que la degradación del alginato es lenta y difícil de controlar [70]. Esto supone un problema, dado que para que haya una buena regeneración tisular el material debe degradarse y permitir que las células fabriquen su propia matriz extracelular. Además, las propiedades requeridas para la fabricación de distintos tejidos son diferentes, por lo que suele ser necesario combinar el alginato con otros biomateriales para conseguir las propiedades mecánicas y estructurales óptimas para cada caso [69]. Así pues, el alginato combinado con otros biomateriales tiene, hoy en día, un papel fundamental en la regeneración de tejidos, destacando entre ellos el cartílago, hueso [71] y tejido vascular [72]. Uno de los desafíos más importantes a los que nos enfrentamos en este campo es en reducir la brecha bench-to-bedside para una correcta funcionalidad del 
tejido bioimpreso. Para ello, varios grupos de investigación de todo el mundo están trabajando en la incorporación y liberación controlada de factores de crecimiento en construcciones bioimpresas de alginato [73].

\section{OTROS USOS}

En la industria del papel, el alginato proporciona una película continua lisa y una superficie con menos pelusa con mejor capacidad de recubrimiento que otros espesantes y gran calidad de retención de tinta e impresión [74]. El alginato también se usa en adhesivos para fabricar tableros porque estabiliza la viscosidad del adhesivo y permite el control de su velocidad de penetración [75]. Además, el alginato puede utilizarse en recubrimientos que se aplican a varillas o electrodos de soldadura para controlar las condiciones en las inmediaciones de la soldadura, como la temperatura o la disponibilidad de oxígeno e hidrogeno [76]. Por último, con el objetivo de finalizar esta lista infinita de aplicaciones industriales que ofrece este valioso biopolímero, cabe mencionar que se han propuesto recientemente adhesivos de alginato de sodio como aglutinantes de fibras de madera/fibras residuales textiles para el aislamiento de edificios [77].

\section{CONCLUSIONES}

Como se ha demostrado en el presente trabajo de revisión, las aplicaciones del alginato son ya inmensas. Sin embargo, las excelentes propiedades que presenta este biopolímero hacen que cada día surjan nuevas aplicaciones tanto en biomedicina como en una gran multitud de campos científicos avanzados. El alginato es un material no tóxico, biodegradable y soluble en agua que, como se ha mencionado, puede obtenerse de fuentes renovables como las algas pardas o cultivos microbianos. Por tanto, es un material que cumple con todos los principios de la química verde y desarrollo sostenible. A continuación, se muestra una tabla resumen de las principales aplicaciones del alginato. 
Tabla 1. Resumen de las inmensas aplicaciones del biopolímero de alginato

\begin{tabular}{|c|c|c|}
\hline Área & Uso específico & Referencia \\
\hline \multirow{7}{*}{$\begin{array}{l}\text { Biotecnología, bioingeniería, } \\
\text { biomedicina y clínica }\end{array}$} & Apósitos para heridas y quemaduras & [78] \\
\hline & Quelante de metales pesados & [61] \\
\hline & Andamiaje en ingeniería tisular & [79] \\
\hline & Liberación controlada & [80] \\
\hline & Bioimpresión 3D & [68] \\
\hline & Prótesis & {$[56]$} \\
\hline & Inmovilización de enzimas y células & [81] \\
\hline \multirow{2}{*}{ Industria farmacéutica } & Complementos alimenticios & [82] \\
\hline & Tratamiento para reflujo gástrico & [62] \\
\hline \multirow{6}{*}{$\begin{array}{l}\text { Industria química, textil } \\
\text { y de la construcción }\end{array}$} & Cosmética & {$[4]$} \\
\hline & Tintas textiles & [83] \\
\hline & Detergentes & {$[8]$} \\
\hline & Adhesivos & [75] \\
\hline & Soldaduras & [76] \\
\hline & Aislamiento de edificios & [77] \\
\hline \multirow{4}{*}{ Alimentos y bebidas } & Fabricación de helados & [21] \\
\hline & Aglutinante y espesante & [32] \\
\hline & Estabilizador de espuma de cerveza & [84] \\
\hline & Confitería y gastronomía en general & [27] \\
\hline Acuicultura & Aglutinante para alimentación & [85] \\
\hline Odontología & Moldes & [86] \\
\hline Industria del papel & Espesante & [74] \\
\hline Arte y artesanía & Moldes de taxidermia & [87] \\
\hline Industria del ocio & Máscaras protectoras & {$[88]$} \\
\hline
\end{tabular}

\section{AGRADECIMIENTOS}

Los autores agradecen a la Fundación Universidad Católica de Valencia San Vicente Mártir el apoyo económico a través del proyecto 2019-231-003UCV.

\section{REFERENCIAS BIBLIOGRÁFICAS}

[1] Serrano-Aroca Á, Ruiz-Pividal JF, Llorens-Gámez M, Enhancement of water diffusion and compression performance of crosslinked alginate with a minuscule amount of graphene oxide, Sci. Rep. 2017;7:11684. DOI: 10.1038/s41598-017-10260-x. 
[2] Lee KY, Mooney DJ. Alginate: Properties and biomedical applications, Prog. Polym. Sci. 37. 2012:106-26. DOI: 10.1016/j.progpolymsci.2011.06.003.

[3] Jeon O, Bouhadir KH, Mansour JM, Alsberg E. Photocrosslinked alginate hydrogels with tunable biodegradation rates and mechanical properties, Biomaterials. 2009;30:2724-34. DOI: 10.1016/j.biomaterials.2009.01.034.

[4] Ethier A, Bansal P, Baxter J, Langley N. The Role of Excipients in the Microstructure of Topical Semisolid Drug Products, n.d. DOI: 10.1007/978-3-030-17355-5.

[5] Fu S, Thacker A, Sperger DM, Boni RL, Velankar S, Munson EJ, Block LH. Rheological evaluation of inter-grade and inter-batch variability of sodium alginate, AAPS PharmSciTech. 2010;11:1662-74. DOI: 10.1208/s12249-010-9547-0.

[6] Efentakis M, Buckton G. The effect of erosion and swelling on the dissolution of theophylline from low and high viscosity sodium alginate matrices, Pharm. Dev. Technol. 2002;7:69-77. DOI: $10.1081 /$ PDT-120002232.

[7] Fertah M. Isolation and Characterization of Alginate from Seaweed, Elsevier Inc.; 2017. DOI: 10.1016/B978-0-12-809816-5.00002-5.

[8] Chan AWJ, Mazeaud I. Becker T. Neufeld RJ. Granulation of subtilisin by internal gelation of alginate microspheres for application in detergent formulation, Enzyme Microb. Technol. 2006;38:265-72. DOI: 10.1016/j.enzmictec.2005.08.018.

[9] Yamamoto T. R.U.S.A. Data, United States Patent. 1997;19.

[10] Yuen CWM, Ku SKA, Choi PS, Kan CW. The effect of the pretreatment print paste contents on colour yield of an ink-jet printed cotton fabric, Fibers Polym. 2004;5:117-21. DOI: 10.1007/ BF02902924.

[11] Abdel-Halim ES, Emam HE, El-Rafie MH. Utilization of hydroxypropyl cellulose and poly(acrylic acid)-hydroxypropyl cellulose composite as thickeners for textile printing, Carbohydr. Polym. 2008;74:938-41. DOI: 10.1016/j.carbpol.2008.05.013.

[12] Perrin Akcakoca Kumbasar E, Bide M. Reactive dye printing with mixed thickeners on viscose. Dye. Pigment. 2000;47:189-99. DOI: 10.1016/S0143-7208(00)00075-9.

[13] Heliopoulos NS, Papageorgiou SK, Galeou A, Favvas EP, Katsaros FK, Stamatakis K. Effect of copper and copper alginate treatment on wool fabric. Study of textile and antibacterial properties. Surf. Coatings Technol. 2013;235:24-31. DOI: 10.1016/j.surfcoat.2013.07.009.

[14] Mihailović D, Šaponjić Z, Radoičić M, Radetić T, Jovančić P, Nedeljković J, Radetić M. Functionalization of polyester fabrics with alginates and $\mathrm{TiO} 2$ nanoparticles. Carbohydr. Polym. 2010;79:526-32. DOI: 10.1016/j.carbpol.2009.08.036.

[15] Fan L, Du Y, Huang R, Wang Q, Wang X, Zhang L. Preparation and characterization of alginate/ gelatin blend fibers, J. Appl. Polym. Sci. 2005;96:1625-9. DOI: 10.1002/app.21610.

[16] Niekraszewicz B, Niekraszewicz A. The structure of alginate, chitin and chitosan fibres, Woodhead Publishing Limited. 2009. DOI: 10.1533/9781845697310.2.266.

[17] Petrulyte S. Advanced textile materials and biopolymers in wound management. Dan. Med. Bull. 2008;55:72-7.

[18] Herrera-Alonso M, McCarthy TJ, Jia X. Nylon surface modification: 2. Nylon-supported composite films, Langmuir. 2006;22:1646-51. DOI: 10.1021/la0526737. 
[19] Corona-Hernandez RI, Álvarez-Parrilla E, Lizardi-Mendoza J, Islas-Rubio AR, de la Rosa LA, Wall-Medrano A. Structural stability and viability of microencapsulated probiotic bacteria: A review. Compr. Rev. Food Sci. Food Saf. 2013;12:614-28. DOI: 10.1111/1541-4337.12030.

[20] Sheu TY, Marshall RT. Microentrapment of Lactobacilli in Calcium Alginate Gels. J. Food Sci. 1993;58:557-61. DOI: 10.1111/j.1365-2621.1993.tb04323.x.

[21] Bahramparvar M, Tehrani MM. Application and functions of stabilizers in ice cream. Food Rev. Int. 2011;27:389-407. DOI: 10.1080/87559129.2011.563399.

[22] Cook DJ, Hollowood TA, Linforth RST, Taylor AJ. Correlating instrumental measurements of texture and flavour release with human perception. Int. J. Food Sci. Technol. 2005;40:631-41. DOI: $10.1111 / \mathrm{j} .1365-2621.2005 .00973 . x$.

[23] Regand A, Goff HD. Structure and ice recrystallization in frozen stabilized ice cream model systems. Food Hydrocolloids. 2003;17:95-102. https://doi.org/10.1016/S0268-005X(02)00042-5.

[24] Probst Y. A review of the nutrient composition of selected rubus berries. 2015. DOI: 10.1108/ NFS-07-2014-0063.

[25] Kothalawala SG, Sivakumaran K. An overview of nanotechnology applications in food industry. Int. J. Sci. Res. Publ. 2018;8:35-40. DOI: 10.29322/ijsrp.8.7.2018.p7954.

[26] This H. Molecular gastronomy is a scientific discipline, and note by note cuisine is the next culinary trend, Flavour. 2013;2: 1-8. DOI: 10.1186/2044-7248-2-1.

[27] Lee P, Rogers MA. Effect of calcium source and exposure-time on basic caviar spherification using sodium alginate. Int. J. Gastron. Food Sci. 2012;1:96-100. DOI: 10.1016/j.ijgfs.2013.06.003.

[28] Aguilera JM. Relating Food Engineering to Cooking and Gastronomy. Compr. Rev. Food Sci. Food Saf. 2018;17:1021-39. DOI: 10.1111/1541-4337.12361.

[29] Tsai FH, Chiang PY, Kitamura Y, Kokawa M, Islam MZ. Producing liquid-core hydrogel beads by reverse spherification: Effect of secondary gelation on physical properties and release characteristics. Food Hydrocoll. 2017;62:140-8. DOI: 10.1016/j.foodhyd.2016.07.002.

[30] Yuasa M, Tagawa Y, Tominaga M. The texture and preference of "mentsuyu (Japanese noodle soup base) caviar" prepared from sodium alginate and calcium lactate. Int. J. Gastron. Food Sci. 2019;18:100178. https://doi.org/10.1016/j.ijgfs.2019.100178.

[31] Sa G, Aa K, Tr S. Characterization of flavoured sweet water balls prepared by basic spherification technique, 2019;7:1714-8.

[32] Y. Qin, J. Jiang, L. Zhao, J. Zhang, F. Wang, Food Ingredient, Elsevier Inc., 2018. DOI: 10.1016/ B978-0-12-811449-0/00013-X.

[33] P. Sriamornsak, Preliminary investigation of some polysaccharides as a carrier for cell entrapment, Eur. J. Pharm. Biopharm. 46 (1998) 233-6. DOI: 10.1016/S0939-6411(98)00021-6.

[34] Manolov RJ, Kambourova MS, Emanuilova EI. Immobilization of Bacillus stearothermophilus Cells by Entrapment in Various Matrices, Process Biochem. 1995;30:141-4. DOI: 10.1016/00329592(95)80004-2.

[35] Taha Ibrahim Zaghloul, Ahmad Rafik EL-Mahdy, Mohammad Hisham EL-Massry, Ben Amar Cheba. Enhanced Production of Bacillus sp. R2 Chitinase through Cell Immobilization. 2011: 8-13.

[36] Pierre AC. The sol-gel encapsulation of enzymes, Biocatal. Biotransformation. 2004;22:145-70. DOI: $10.1080 / 10242420412331283314$. 
[37] Ferrer P, Solà C. Lipase production by immobilized Candida rugosa cells. Appl. Microbiol. Biotechnol. 1992;37:737-41. DOI: 10.1007/BF00174838.

[38] Chakraborty S. Carrageenan for encapsulation and immobilization of flavor, fragrance, probiotics, and enzymes: A review. J. Carbohydr. Chem. 2017;36:1-19. DOI: 10.1080/07328303.2017.1347668.

[39] Marpani F, Luo J, Mateiu RV, Meyer AS, Pinelo M. In Situ Formation of a Biocatalytic Alginate Membrane by Enhanced Concentration Polarization,ACS Appl. Mater. Interfaces. 2015;7:17682-91. DOI: 10.1021/acsami.5b05529.

[40] Xu SW, Lu Y, Li J, Jiang ZY, Wu H. Efficient conversion of CO 2 to methanol catalyzed by three dehydrogenases co-encapsulated in an alginate-silica (ALG-SiO 2 ) hybrid gel, Ind. Eng. Chem. Res. 2006;45:4567-73. DOI: 10.1021/ie0514071.

[41] Taqieddin E, Amiji M. Enzyme immobilization in novel alginate-chitosan core-shell microcapsules, Biomaterials. 2004;25:1937-45. DOI: 10.1016/j.biomaterials.2003.08.034.

[42] Blandino A, Macías M, Cantero D. Immobilization of glucose oxidase within calcium alginate gel capsules, Process Biochem. 2001;36:601-6. DOI: 10.1016/S0032-9592(00)00240-5.

[43] Subramaniam A, Reilly A. Preparation of microcapsules. 2004;4.

[44] Palmieri G, Giardina P, Desiderio B, Marzullo L, Giamberini M, Sannia G. A new enzyme immobilization procedure using copper alginate gel: Application to a fungal phenol oxidase. Enzyme Microb. Technol. 1994;16:151-8. DOI: 10.1016/0141-0229(94)90078-7.

[45] Beris AE, Lykissas MG, Papageorgiou CD, Georgoulis AD. Advances in articular cartilage repair. 2005:14-23. DOI: 10.1016/j.injury.2005.10.007.

[46] Spermvital. http://www.spermvital.com/. [Consultado: 30 de enero de 2020].

[47] Raizada K, Rani D. Ocular prosthesis. Contact Lens Anterior Eye. 2007;30:152-62. DOI: 10.1016/j.clae.2007.01.002.

[48] Liu W, Griffith M, Li F. Alginate microsphere-collagen composite hydrogel for ocular drug delivery and implantation. J. Mater. Sci. Mater. Med. 2008;19:3365-71. DOI: 10.1007/s10856008-3486-2.

[49] Silva D, Pinto LFV, Bozukova D, Santos LF, Serro AP, Saramago B. Chitosan/alginate based multilayers to control drug release from ophthalmic lens. Colloids Surfaces B Biointerfaces. 2016;147:81-9. DOI: 10.1016/j.colsurfb.2016.07.047.

[50] Kulkarni MM, Thombare RU. Dimensional changes of alginate dental impression materials-an invitro study. J. Clin. Diagnostic Res. 2015;9:ZC98-ZC102. DOI: 10.7860/ JCDR/2015/13627.6407.

[51] Inoue K, Song YX, Fujii K, Kadokawa A, Kanie T. Consistency of alginate impression materials and their evaluation. J. Oral Rehabil. 1999;26:203-7. DOI: 10.1046/j.1365-2842.1999.00382.x.

[52] Cook W. Alginate dental impression materials: Chemistry, structure, and properties. J. Biomed. Mater. Res. 1986;20:1-24. DOI: 10.1002/jbm.820200103.

[53] Kanafi MM, Ramesh A, Gupta PK, Bhonde RR. Dental pulp stem cells immobilized in alginate microspheres for applications in bone tissue engineering. Int. Endod. J. 2014(47):687-97. DOI: 10.1111/iej.12205.

[54] Moshaverinia A, Chen C, Akiyama K, Ansari S, Xu X, Chee WW, Schricker SR, Shi S. Alginate hydrogel as a promising scaffold for dental-derived stem cells: An in vitro study. J. Mater. Sci. Mater. Med. 2012;23:3041-51. DOI: 10.1007/s10856-012-4759-3. 
[55] Yamamoto Y, Mendel E, Raffel C. Acrylic cranioplasty with alginate molding: Technical note, Neurosurgery. 1997;41:305-7. DOI: 10.1097/00006123-199707000-00055.

[56] Blind A, Hulterström A, Berggren D. Treatment of nasal septal perforations with a custom-made prosthesis. Eur. Arch. Oto-Rhino-Laryngology. 2009;266:65-9. DOI: 10.1007/s00405-0080690-0.

[57] Eaton LD, Dryden RM, Popp JC, Harner D. Functional Anatomical Reconstruction in an Oculofacial Prosthesis. Ophthalmology. 1984;91: 984-6. DOI: 10.1016/S0161-6420(84)34205-1.

[58] Purcell EK, Seymour JP, Yandamuri S, Kipke DR. In vivo evaluation of a neural stem cell-seeded prosthesis. J. Neural Eng. 2009;6:026005. DOI: 10.1088/1741-2560/6/2/026005.

[59] Cervino G, Fiorillo L, Herford AS, Laino L, Troiano G, Amoroso G, Crimi S, Matarese M, D'Amico C, Siniscalchi EN, Cicciù M. Alginate materials and dental impression technique: A current state of the art and application to dental practice. Mar. Drugs. 2019;17:1-15. DOI: 10.3390/md17010018.

[60] Sun J, Wei D, Yang K, Yang Y, Liu X, Fan H, Zhang X. The development of cell-initiated degradable hydrogel based on methacrylated alginate applicable to multiple microfabrication technologies. J. Mater. Chem. B. 2017;5:8060-9. DOI: 10.1039/c7tb01458a.

[61] Eliaz I, Weil E, Wilk B. Integrative medicine and the role of modified citrus pectin/alginates in heavy metal chelation and detoxification - Five case reports, Forsch. Komplementarmed. 2007; 14:358-64. DOI: 10.1159/000109829.

[62] Baldassarre ME, Di Mauro A, Pignatelli MC, Fanelli M, Salvatore S, Di Nardo G, Chiaro A, Pensabene L, Laforgia N. Magnesium Alginate in Gastro-Esophageal Reflux: A Randomized Multicenter Cross-Over Study in Infants. Int J Environ Res Public Health. 2020;17:83.

[63] Langer R. Biomaterials in Drug Delivery and Tissue Engineering: One Laboratory's. Experience. 2000;33:94-101.

[64] Li C, Vepari C, Jin H, Joo H, Kaplan DL. Electrospun silk-BMP-2 scaffolds for bone tissue engineering. 2006;27:3115-24. DOI: 10.1016/j.biomaterials.2006.01.022.

[65] Richmon JD, Sage AB, Shelton E, Schumacher BL, Sah RL, Watson D. Effect of Growth Factors on Cell Proliferation, Matrix Deposition, and Morphology of Human Nasal Septal Chondrocytes Cultured in Monolayer. 2005:1553-60.

[66] AlgiPharma. https://algipharma.com/. [Consultado: 30 de enero de 2020].

[67] Axpe E, Oyen ML. Applications of alginate-based bioinks in 3D bioprinting. Int. J. Mol. Sci. 2016;17. DOI: 10.3390/ijms17121976.

[68] Kang HW, Lee SJ, Ko IK, Kengla C, Yoo JJ, Atala A. A 3D bioprinting system to produce human-scale tissue constructs with structural integrity. Nat. Biotechnol. 2016;34:312-9. DOI: $10.1038 / \mathrm{nbt} .3413$.

[69] Hölzl K, Lin S, Tytgat L, Van Vlierberghe S, Gu L, Ovsianikov A. Bioink properties before, during and after 3D bioprinting, Biofabrication. 2016;8: DOI: 10.1088/1758-5090/8/3/032002.

[70] Jia J, Richards DJ, Pollard S, Tan Y, Rodriguez J, Visconti RP, Trusk TC, Yost MJ, Yao H, Markwald RR, Mei Y. Engineering alginate as bioink for bioprintin. Acta Biomater. 2014;10:432331. DOI: $10.1016 / j$.actbio.2014.06.034.

[71] Wüst S, Godla ME, Müller R, Hofmann S. Tunable hydrogel composite with two-step processing in combination with innovative hardware upgrade for cell-based three-dimensional bioprinting. Acta Biomater. 2014;10:630-40. DOI: 10.1016/j.actbio.2013.10.016. 
[72] Zhang Y, Yu Y, Chen H, Ozbolat IT. Characterization of printable cellular micro-fluidic channels for tissue engineering. Biofabrication. 2013;5:1-23. DOI: 10.1088/1758-5082/5/2/025004.

[73] Poldervaart MT, Wang H, Van Der Stok J, Weinans H, Leeuwenburgh SCG, Oner FC, Dhert WJA, Alblas J. Sustained release of BMP-2 in bioprinted alginate for osteogenicity in mice and rats. PLoS One. 2013;8. DOI: 10.1371/journal.pone.0072610.

[74] Rinaudo M. Biomaterials based on a natural polysaccharide: alginate. TIP. 2014;17:92-6.

[75] Bilbao-Sainz C, Chiou BS, Glenn GM, Orts WJ. Biobased adhesives, gums, emulsions, and binders: current trends and future prospects. Journal of Adhesion Science and Technology. 2013;27:1972-97

[76] Dickinson BBE. Food Colloids: Proteins, Lipids and Polysaccharides. Woodhead publishing limited. Cambridge. 2004.

[77] Lacostea C, El Hage R, Bergeret A, Corna S, Lacroixc P. Sodium alginate adhesives as binders in wood fibers/textile waste fibers biocomposites for building insulation. Carbohydrate Polymers. 2018;184:1-8

[78] Hunt NC, Shelton RM, Grover LM. An alginate hydrogel matrix for the localised delivery of a fibroblast/keratinocyte co-culture. Biotechnol. J. 2009;4:730-7. DOI: 10.1002/biot.200800292.

[79] Sun J, Tan H. Alginate-Based Biomaterials for Regenerative Medicine Applications. 2013:1285309. DOI: $10.3390 / \mathrm{ma} 6041285$.

[80] Silva D, Pinto LFV, Bozukova D, Santos LF, Serro AP, Saramago B. Chitosan/alginate based multilayers to control drug release from ophthalmic lens. Colloids Surfaces B Biointerfaces. 2016;147:81-9. https://doi.org/10.1016/j.colsurfb.2016.07.047.

[81] Andersen T, Auk-emblem P, Dornish M. 3D Cell Culture in Alginate Hydrogels. 2015:133-61. DOI: $10.3390 /$ microarrays4020133.

[82] Suleria HA, Osborne S, Gobe G. Marine-based Nutraceuticals: An innovative Trend in the Food and Supplement Industries. 2015:6336-51. DOI: 10.3390/md13106336.

[83] Akira M, Luis D. Rheological evaluation of Laponite / alginate inks for 3D extrusion-based printing; 2018.

[84] Sugawara E, Nikaido H. Alginates: Biology and Applications, Antimicrob. Agents Chemother. 2009;13:7250-7. DOI: 10.1007/978-3-540-92679-5.

[85] Storebakken T. Binders in fish feeds: I. Effect of alginate and guar gum on growth, digestibility, feed intake and passage through the gastrointestinal tract of rainbow trout. Aquaculture. 1985;47:11-26.

[86] Lan S, Kehinde T, Zhang X, Khajotia S, Schmidtke DW, Starly B. Controlled release of metronidazole from composite poly- $\varepsilon$-caprolactone / alginate ( PCL / alginate ) rings for dental. Dent. Mater. 2013:1-10. DOI: 10.1016/j.dental.2013.03.014.

[87] Burhouse S, Hartman TPV. Casts of fluid preserved specimens. Methods Mol. Biol. 2019;1897:155-62. DOI: 10.1007/978-1-4939-8935-5_16.

[88] Cazon A, Aizpurua J, Paterson A, Bibb R, Campbell RI. Customised design and manufacture of protective face masks combining a practitioner-friendly modelling approach and low-cost devices for digitising and additive manufacturing: This paper analyses the viability of replacing conventional practice with AM method to make customized protective face masks. Virtual Phys. Prototyp. 2014;9:251-61. DOI: 10.1080/17452759.2014.958648. 
